\title{
Cardiac pathology and modern therapeutic approach in Behçet disease
}

\author{
Giuseppe Cocco, Paul Jerie \\ Cardiology Office, Rheinfelden, Switzerland
}

\begin{abstract}
Behçet disease $(B D)$ is an enigmatic inflammatory disorder with multisystemic complications which is endemic in some countries but can be seen in the entire world. Valid diagnostic criteria are available. The pathology is related to a specific perivasculitis with involvement of both arteries and veins of all sizes. Minor arterial and cardiac involvement is frequent in BD but is usually asymptomatic. In exceptional cases cardiac symptoms may be the $1^{\text {st }}$ manifestation of BD. The prevalence of severe cardiac complications (cardio-Behçet) should be $<10 \%$. An impressive therapeutic improvement has been achieved by using appropriate catheterization techniques, coronary and intra-arterial stents, colchicine, drug-response modifying drugs and immunotherapy but, still cardio-Behçet has a poor prognosis. Efforts are undertaken to improve morbidity and prognosis with the use of newer drugs. An important part of the complications in $B D$ are related to the frequent thromboembolic complications and there is high possibility that newer oral anticoagulants will be superior to the classical anticoagulants presently used. Available biologic agents have already been frequently used and seem to have improved the prognosis, but efforts are undertaken to find newer biologic agents with better therapeutic performance and less side-effects.

Summarizing as much as possible the effects of the presently used biotherapy in $B D$, interferon- $\alpha$ is effective against many ocular, genital and perhaps vascular manifestations, but its effectiveness is limited by frequent adverse-effects (even if not dangerous for the cardiovascular system). Infliximab is a valid option in the therapy of ocular and cutaneous manifestations but it is less convincing in the therapy of vascular manifestations in vascular-and neuro-Behçet; furthermore, side-effects, including severe cardiovascular complications, are seen in a minority of patients; perhaps worse, infliximab seems to loose efficacy in the long-term therapy, while pharmacogenetics and receptor polymorphism may explain the existence of non-responders and the occurrence of resistance. Adalimumab might be a promising alternative for infliximab and seems to exert a good effect in aneurysmatic and other vascular complications. However, we lack long-term studies. Other biologic agents have been used only in few cases and it is too early to say if they offer new therapeutic perspectives. (Cardiol J 2014; 21, 2: 105-114)
\end{abstract}

Key words: Behçet disease, cardio-Behçet, novel oral anticoagulants, immunotherapy, biotherapy

Address for correspondence: Giuseppe Cocco, MD, FESC, Cardiology Office, Marktgasse 10A, POB 119; CH-4310 Rheinfelden, Switzerland, e-mail: praxis@cocco.ch 


\section{Introduction}

The terms "Behçet disease", "vasculo-Behçet", "cardio-Behçet", "therapy of Behçet disease with novel oral anticoagulants" and "immunotherapy in Behçet disease" were used for a search through MEDLINE, EMBASE, Scopus, and Web of Science. Thousands of references were found and we used the most important to support our experience with the pathology. Our review describes the impact of Behçet disease (BD) in frequent (mild) and (rare) severe vascular and cardiac complications of BD (cardio-Behçet). The therapeutic impact and value of novel oral anticoagulants and biologic agents is also discussed.

\section{History and epidemiology}

$\mathrm{BD}$ is a chronic, relapsing, multisystemic disorder, and its historical descriptions can be found in the works of Hippocrates and ancient traditional Chinese medicine [1]. Adamantiades in 1930 and 1931 published the pathology of 3 patients with relapsing iritis, hypopyon, leg ulcerations and thrombophlebitis and stated that this was a new nosological entity; however, the pathology became really known in 1937 (and since then has been called BD) when Behçet also published a paper on patients with a triad of recurrent oral and genital aphthous ulcerations [2]. BD clusters along the ancient Silk Road [3], which extends from eastern Asia to the Mediterranean basin, but cases are detected in the entire world and not exclusively in the migrant population [4].

\section{Diagnosis and classification}

The International Study Group (ISG) [5] defined 5 diagnostic criteria: 2 are mucous membrane manifestations, i.e. oral and genital aphthosis, the $3^{\text {rd }}$ is skin manifestations (pseudofolliculitis and erythema nodosum), the $4^{\text {th }}$ is ocular manifestations (anterior and/or posterior uveitis and retinal vasculitis) and the $5^{\text {th }}$ is the pathergy phenomenon. In the ISG classification the presence of oral aphthosis is mandatory and 2 of the remaining items (genital aphthosis, skin, eye, pathergy phenomenon) are necessary to acknowledge existence of BD. In 2005, 27 multinational experts used large numbers of patients and controls with different ethnicities and created the International Criteria for $\mathrm{BD}$ (ICBD) [6]. In the ICBD classification 6 criteria are used: 1) mucous (oral and genital) aphthosis, 2) skin lesions (pseudofolliculitis, erythema nodosum), 3) eye lesions (anterior and/or posterior uveitis, retinal vasculitis), 4) vascular complications, 5) vasculitis, and 6) pathergy phenomenon. In the ICBD classification genital aphthous lesions and eye lesions have bigger diagnostic value than the others and give each 2 points; the other 4 items get 1 point each. In the ICBD the diagnosis of $\mathrm{BD}$ is acknowledged when a patient gets $\geq 3$ points.

\section{Etiology and pathophysiology}

The etiology of BD remains elusive but ethnic background, gender-related factors, HLA markers, T-lymphocytes dysfunction, circulating immune complexes, cytokines, genetically predisposed inflammation and hypercoagulation are thought to be intimately involved in pathophysiology of BD $[1,4]$. The underlying pathophysiology of $\mathrm{BD}$ is a distinctive perivasculitis with involvement of both arteries and veins of all sizes [7]. Unlike other systemic vasculitides and autoimmune diseases, the perivasculitis of $\mathrm{BD}$ is not associated with anti-Ro, antiphospholipid and antineutrophil cytoplasmic antibodies [8]. In BD pathological thrombosis can be best viewed within the classical Virchow's triad. Vascular complications are: 1) venous and 2) arterial thrombosis, 3) arterial aneurysm, 4) combined arterial thrombosis and aneurysm, and 5) combined venous thrombosis and arterial lesions [7]. It is thought that perivascular structures are the main target of T-lymphocytes mediated immune reactions [9]. The venous and arterial wall lesions attract cytokinergic and neutrophilic reactions. It is thought [10] that the interleukin-23/ /interleukin-17 axis should play a role in inducing $\mathrm{BD}$ (the interleukin-17 cytokine family consists of 6 cytokines, interleukins from 17A to 17F, and 5 receptors, interleukins from 17RA to 17RE). Neutrophils infiltrate the vessels and are activated, inducing destructive effects by overproduction of superoxide anion radicals and lysosomal enzymes, promoting advanced vascular wall destruction with aneurysm formation, and in the end causing local blood flow abnormalities. Macrophages play an important pathological role at sites of plaque rupture in the thrombosed coronary arteries of patients with acute myocardial infarction [11]. It is known that apolipoprotein B - containing lipoproteins, transforms monocytes into macrophages which in turn drive the progression of arterial inflammatory lesions [12]. Indeed, monocytes and some macrophages can be detected in the involved arterial walls of vascular- and cardio-Behçet patients and it is logical to assume that they contribute to the complications in these vessels. Disturbed flow ge- 
Table 1. Asymptomatic cardiac manifestations in Behçet disease, and symptomatic cardiac manifestations (cardio-Behçet).

\begin{tabular}{ll}
\hline A. Detected by electrocardiography & \\
\hline Asymptomatic manifestations & Cardio-Behçet \\
\hline AV-block grade I (or grade II Wenckenbach) & AV-block > grade II Mobitz type II \\
Asymptomatic premature beats & Symptomatic premature beats \\
Asymptomatic brady-/tachycardia & Symptomatic brady-tachycardia \\
Asymptomatic atrial fibrillation & Symptomatic atrial fibrillation \\
Right or left ventricular block & Arrhythmias induced by long QT and \\
Prolonged QT and abnormal late potentials & or abnormal late potentials \\
& Myocardial ischemia \\
\hline B. Detected by echocardiography, magnetic resonance imaging and computed tomography \\
\hline Asymptomatic manifestations & Infarction \\
\hline Non occlusive pericarditis & Cardio-Behçet \\
Inter-atrial septal aneurysm & Constrictive pericarditis \\
Aneurysmatic enlargement of sinus valsalve & Mitral prolapse without regurgitation \\
and ascending aorta & Ventricular aneurysms and thrombi \\
Coronary aneurysms atrial enlargement & Endocarditis with valvular dysfunction \\
Mild systolic or diastolic dysfunction & Floppy mitral valve \\
Minor contractile dysfunction (TDI) & Major aortic regurgitation \\
Endocarditis without valvular dysfunction & Severe systolic dysfunction \\
Endomyocardial fibrosis & \\
Intra-cardiac aneurysms and thrombi & \\
Minor aortic regurgitation & \\
Mitral prolapse with regurgitation & \\
\hline
\end{tabular}

nerates shear stress and consequently stimulates specific mechanosensors located on the surface of endothelial cells and consequently pro-atherogenic genes are up-regulated and athero-protective genes are down-regulated. Furthermore, in patients with $\mathrm{BD}$, one also finds elevation in some markers of endothelial dysfunction (thrombomodulin and adhesion molecules) and low-grade inflammation (proinflammatory cytokines, C-reactive protein, and serum amyloid A), confirming their important role in pathophysiology of most immune-mediated cardiovascular events in this pathology [13]. Finally, in the perivasculitis of BD endothelial dysfunction, release of von Willebrand factor, platelet activation, enhanced thrombin and fibrin generation, antithrombin deficiency and impaired fibrinolysis close the pathologic chain of enhanced thrombocoagulation [13]. Depending on the organs affected by the vascular lesions there is the clinical occurrence of a vascular-, neuro-, or cardio-Behçet.

Whatever the complex and still incompletely understood mechanisms of the perivasculitis, venous pathology and thrombotic complications are hallmarks of $\mathrm{BD}$, recurrent thrombophlebitis is the commonest vascular affection in $\mathrm{BD}$ and venous involvement is reported in almost one third of patients, and arterial involvement in $15 \%$ of cases $[1,4,7,13]$.

\section{Prevalence of cardio-Behçet}

It was recognized that cardiac and arterial complications occur and are an important part in the complications of BD [1, 4, 7, 14-16]. Cardiac involvement in $\mathrm{BD}$ is frequent but is usually mild and asymptomatic. In countries with endemic BD, physicians are familiar with the pathology and are inclined to get a cardiological opinion. In referred patients electrocardiography, echocardiography and, when used, computed tomography or magnetic resonance imaging analysis detect subtle cardiac changes in about one third of cases (they are listed in Table 1). It is, therefore, assumed that minor cardiac complications occur in around a third of patients with BD [1, 14-17]. As in other systemic complications, which are part of the 
diagnostic criteria, the cardiac pathology of BD is not specific. However, the pathology is considered a complication of $\mathrm{BD}$ when biopsies show the typical and specific perivasculitis and also when these complications are detected in patients with $\mathrm{BD}$ without an alternative etiology. While minor cardiac complications are rarely published, the rare cases of cardio-Behçet are interesting and often published [1, 17-22], and this situation may give the wrong impression that cardio-Behçet is not uncommon. In the literature the frequency of cardio-Behçet varies between $7 \%$ and $46 \%$ and this large difference shows that the real prevalence is unknown. Several reasons contribute to different statistics: a) definitions used for cardio-Behçet are not standardized; b) important national differences, and in countries with endemic experienced physicians detect cardiac complications more often than physicians where $\mathrm{BD}$ is rare; c) cardio-Behçet is really more frequent in some ethnicities $[1,4,14$, 23]; and 4) referring habits, because if patients are sent to hospitals there are mandatory statistics, but if they are sent to private cardiologists the cases are rarely found in official statistics. Our group has a long experience with a small number of patients, mostly with migrant origins [1] and centers with many cases $[18,22,23]$ have seen cardio-Behçet in $<10 \%$ of referred patients with BD.

\section{Complications and prognosis in vasculo-Behçet and cardio-Behçet}

The non-cardiac complications of BD were described elsewhere [4]. As shown in Table 1, pericarditis, myocardial involvement (diastolic and/or systolic dysfunction), valvular and coronary complications (thrombosis, aneurysms, and rupture), predominantly right-sided intra-cardiac thrombi and ischemia are the most frequent cardiac manifestations [1, 15-23]. Good examples of cardio-Behçet can be found in our previous paper [1].

Saadoun et al. [23] describe data from 101 $(12.3 \%)$ patients with vasculo-Behçet among a cohort of 820 patients with BD. Arterial lesions included aneurysms (47.3\%), occlusions $(36.5 \%)$, stenosis $(13.5 \%)$, and aortitis $(2.7 \%)$. Lesions mainly involved the aorta (25\% of vasculo-Behçet, $0.03 \%$ of patients with BD), femoral arteries (22.7\% of vasculo-Behçet, $0.028 \%$ of patients with $\mathrm{BD})$ and pulmonary arteries $(0.2 \%$ of vasculo-Behçet, $0.026 \%$ of patients with BD). Patients with arterial lesions were more frequently male $(91.2 \%$ vs. $62.4 \%$, respectively, $\mathrm{p}=0.017$ ) and had higher rates of venous involvement $(80.4 \%$ vs. $29.8 \%$, re- spectively, $\mathrm{p}<0.001$ ) compared to patients without arterial manifestations. In multivariate analysis, the presence of venous involvement (odds ratio [OR] 0.29, 95\% confidence interval [CI] 0.08-1.11) and arterial occlusive lesions (OR 0.13, 95\% CI $0.01-1.25)$ were negatively associated with complete remission. The 20 -year survival rate was significantly lower in patients with than in those without arterial lesions ( $73 \%$ vs. $89 \%$, respectively, $\mathrm{p}<0.0001)$. The use of immunotherapy (OR 3.38, 95\% CI 0.87-13.23) was associated with the occurrence of complete remission and improved the prognosis and $39(38.6 \%)$ of 101 patients with vasculo-Behçet achieved complete remission.

In the same paper [23] describes data from $52(6 \%)$ cases of cardio-Behçet from a cohort of 807 patients with $\mathrm{BD}$. The duration of disease was $29.3 \pm$ \pm 10.3 years (mean $\pm \mathrm{SD}$ ). Cardiac lesions included pericarditis and thrombosis $(19.2 \%)$, myocardial infarction (17.3\%), endomyocardial fibrosis $(7.7 \%)$ and myocardial aneurysm (1.9\%). Patients with cardio-Behçet were more frequently male $(86.5 \%$ vs. $64.9 \%, \mathrm{p}<0.01)$ and had more arterial $(42.3 \%$ vs. $11.1 \%, \mathrm{p}<0.01)$ and venous lesions (59.6\% vs. $35.8 \%, \mathrm{p}<0.01)$ compared to those without cardiac manifestations. Factors associated with complete remission of cardiac involvement were treatment regimens with oral anticoagulants, colchicine and immunotherapy. The 5 -year survival rate was $83.6 \%$ and $95.8 \%(\mathrm{p}=0.03)$ in patients with and without cardiac involvement, respectively. After a median follow-up of 3 (range 1.75-4.2) years 8 cardio-Behçet patients died, and those 3 deaths were directly related to cardiac involvement. The authors conclude that the prognosis of cardio-Behçet patients is poor and improves with oral anticoagulation, colchicine and immunotherapy. When coronary arteries are affected there is the clinical presentation of an acute coronary syndrome but, unlike from arteriosclerotic pathologies, the coronary pathology occurs in relatively young patients without strong association with classical cardiovascular risk factors $[1,15]$.

Affections of inferior vena cava and aorta bear the worst prognosis for patients with BD. Blood clots can develop in the deep veins of the lower extremity and migrate to the right heart and pulmonary arteries $[17,21]$. In some patients with $\mathrm{BD}$, macrophages accumulate in the arteries and contribute to arterial rupture, formation of an aneurysmatic lesion, and secondary thrombotic and ischemic complications. Destruction of elastic structures of aorta can result in formation of an- 
eurysms and pseudoaneurysms prone to rupture; aneurysms of smaller vessels can also develop, sometimes spontaneously [1,21-23]. The risk of aneurysms and pseudoaneurysms is especially high in patients undergoing major (angioplasty of aorta and its branches, coronary artery bypass grafting) or even minor vascular interventions (arterial puncture) [19, 20, 22, 24]. Special use of catheterization techniques and careful closure of the puncture site, combined with appropriate use of anticoagulants, reduces the incidence of iatrogenic aneurysms, but arterial and coronary artery aneurysms still occur and remain a major challenge in vascular- and cardio-Behçet [19, 24].

Our group has already published a paper on cardio-Behçet [1]. We add two typical cases.

Case 1: 39-year-old male patient with migration origins, without classic cardiovascular risk factors. BD was diagnosed 3 years earlier (mucous, genital manifestations and pathergy phenomenon) and was treated with colchicine and azathioprine. Three weeks earlier, the patient noted the occurrence of fatigue, dyspnea, chest pain, dry cough, dizziness and palpitations. Blood pressure was 92/60 $\mathrm{mm} \mathrm{Hg}$ (8 weeks earlier 130/70 $\mathrm{mm} \mathrm{Hg}$ ) and NT-proB-type natriuretic peptide $>2000 \mathrm{pg} / \mathrm{mL}$; D-dimers and troponin A were normal. As shown in Figure 1, the thorax-computed tomography detected a marked left ventricular enlargement and the ECG detected tachycardia with frequent and repetitive ectopic beats, interpreted as of supraventricular origin with $\mathrm{QRS}$-aberration. The type of arrhythmias was confirmed by a 48 -h dynamic ECG. Coronarography detected insignificant coronary artery lesions and a moderate mitral regurgitation; the left ventricular function was reduced to $40 \%$, with diffusely reduced contraction but without regional dyskinesia. The patient was treated with $\beta$-blockers and diuretics and the cardiac function improved within 3 weeks.

Case 2: 41-year-old male patient without migration origins. BD was diagnosed 8 years earlier (ocular, genital, vascular manifestations and pathergy phenomenon) and was treated with colchicine and methotrexate. He also had moderate dyslipidemia which was treated with atorvastatin. In the previous $48 \mathrm{~h}$, suddenly and several times, he experienced typical resting angina pectoris. Troponin $\mathrm{A}$ and D-dimers were strongly positive; ECG (Fig. 2) showed ischemic ST-changes from $V_{3}$ to $\mathrm{V}_{6}$. Coronarography detected a significant stenosis of the left main coronary artery and, unexpectedly, a chest-computed tomography detected an old large right pulmonary infarction (a typical complication of BD). Under therapy with bivalirudin and nitrates the patients was successfully treated with percutaneous trasluminal coronary angioplasty and implantation of a bare metal stent. The course was complicated by an aneurysmatic complication at the femoral arterial catheterization site (a typical complication of $\mathrm{BD}$ ) which required a special occlusion. Under therapy with aspirin, ischemic changes, and echocardiography detected a normal left ventricular ejection fraction with a small non-transmural infarction and hyperkinesia in the left anterior wall.

\section{Outcomes and therapy}

In the past, lethal outcomes of cardio-Behçet were up to $20 \%$ [1, 4, 15, 22]. It seems that the prognosis of cardio-Behçet improves with the appropriate use of colchicine, disease-modifying agents, adapted catheterization techniques, endovascular stents for aneurysmatic lesions, anticoagulation and immunosuppressive therapy $[22,23]$. Colchicine plays a central role in the therapy of BD [25]. At present we are unaware of published data on the use of novel oral anticoagulants (i.e. direct factor $\mathrm{X}$-inhibitors and thrombin inhibitors) in BD but they are being used off-label and seem to offer several advantages over heparin and vitamin $\mathrm{K}$ antagonists.

The body normally produces low levels of biological response-modifiers in response to infection and disease. Immunotherapy (biotherapy) relies on the use of pharmacologically produced biological response-modifiers (biologic agents). Several biologic agents are used to treat cancer, several autoimmune and viral infectious diseases. The therapeutic use of biologic agents in cardiology, their heterogeneity and cardiovascular safety aspects have been recent reviewed [26]. Table 2 summarizes the biologic agents presently used in the therapy of BD and their mechanism of action. Biotherapy includes monoclonal antibodies, interferon, interleukin-2, and several types of colony-stimulating factors (CSF, GM-colony-stimulating factor and G-colony-stimulating factor). Some biologic agents have a specific potential for binding to certain receptors, an aspect which is related to the genetic basis and polymorphism of the receptors; consequently, they have a largely variable efficacy in individual patients and there is the risk of lack of efficacy (with useless high therapeutic costs) or unexpected severe side-effects (with high cost of iatrogenic effects); for this reason, in Germany, before using some biologic agents in oncology, physicians must obtain a pharmacogenetic tissue test [27, 28]. 


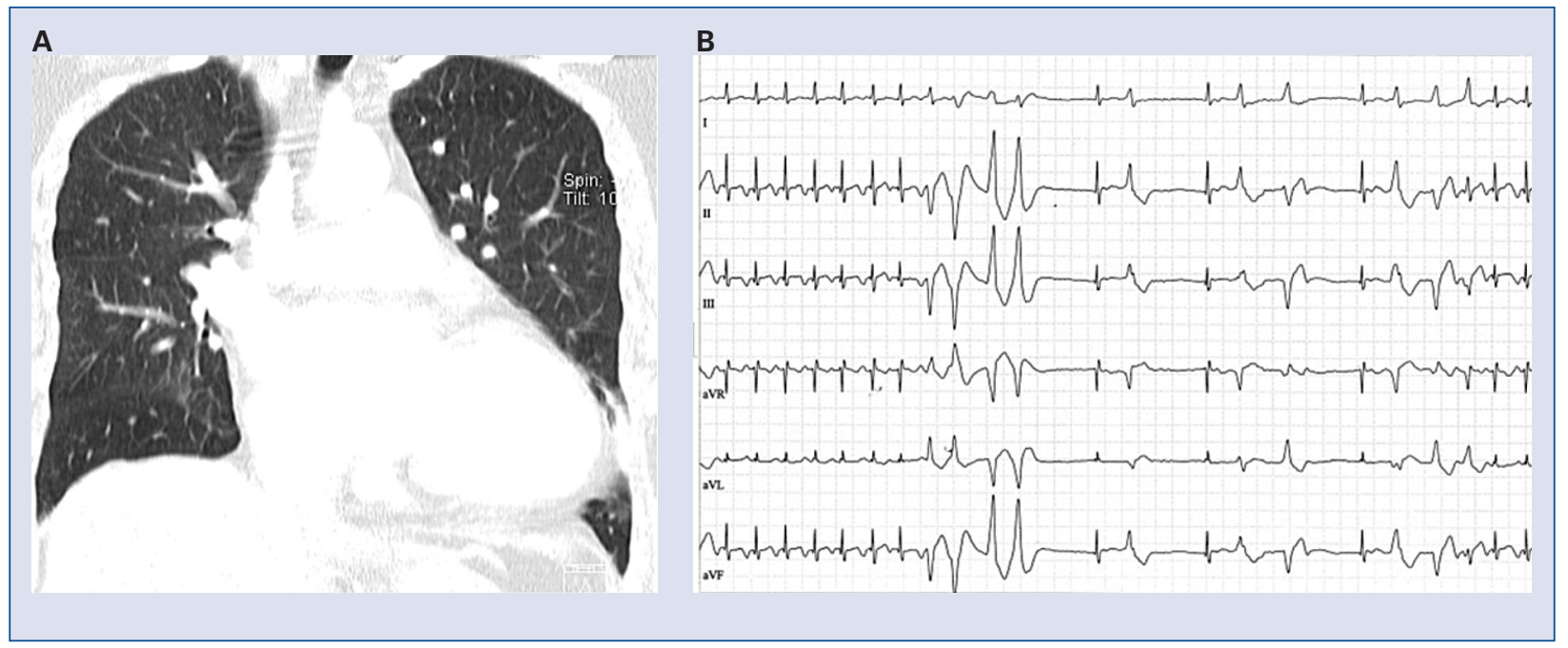

Figure 1. Cardio-Behçet, acute congestive cardiac failure; A. Severe left ventricular enlargement; B. Repetitive supraventricular ectopic beats with QRS aberration.

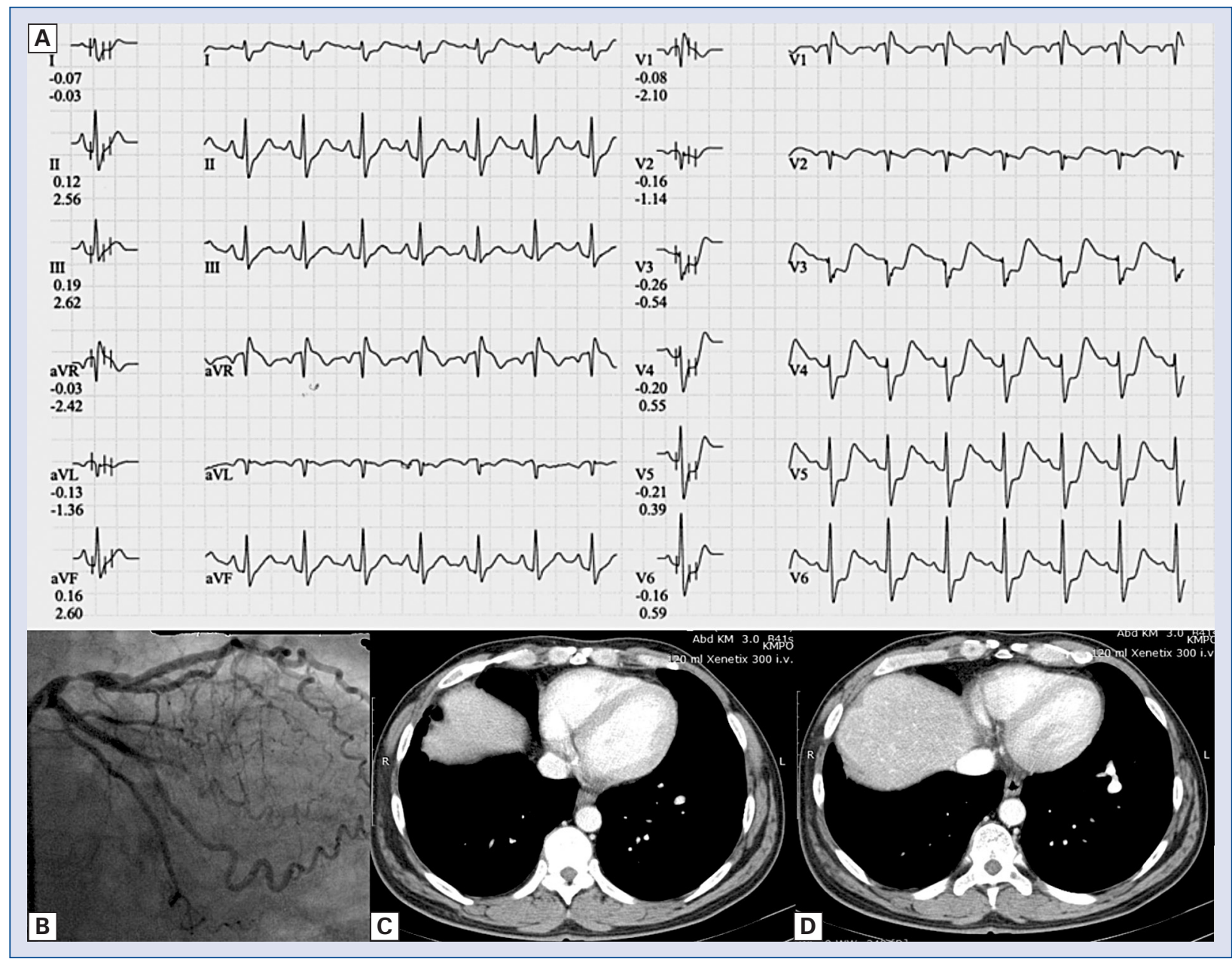

Figure 2. Cardio-Behçet. Acute coronary syndrome with severe occlusion of the left main coronary artery and large right pulmonary infarction; $A$. The ECG shows an ischemic horizontal ST-downsloping in from $\mathrm{V}_{3}$ to $\mathrm{V}_{6}$; $\mathbf{B}$. Coronarography shows a significant occlusion of the left main coronary artery; C, D. Chest-computed tomography shows a large infarction at the basis of right lung from the surface to the proximity of the heart. 
Table 2. Biological agents used in Behçet's disease (BD).

\begin{tabular}{|c|c|c|}
\hline Drug & Class/Action & Use in BD \\
\hline Abatacept & $\begin{array}{l}\text { Fusion protein composed of the Fc region of the } \\
\text { immunoglobulin IgG1, fused to the extracellular } \\
\text { domain of CTLA-4, a molecule capable of binding } \\
\text { B lymphocytes and prevents the delivery } \\
\text { of co-stimulatory signal to T cells to fully activate } \\
\text { them. It also helps to enable down-regulation } \\
\text { of T-cells by way of T-cell anergy }\end{array}$ & Neuro-BD \\
\hline Adalimumab & Fully human monoclonal antibody, TNF inhibitor & Multiple manifestations \\
\hline Anakinra & Interleukin-1 receptor antagonist & Vascular manifestations, neuro-BD \\
\hline Bevacizumab & $\begin{array}{l}\text { Humanized monoclonal antibody that inhibits } \\
\text { vascular endothelial growth factor } A\end{array}$ & Multiple manifestations \\
\hline Brodalumab & $\begin{array}{l}\text { Human monoclonal antibody, } \\
\text { anti-interleukin-17-receptor }\end{array}$ & Multiple manifestations \\
\hline Imatinib & Receptor tyrosine kinase inhibitor & Multiple manifestations \\
\hline Infliximab & $\begin{array}{l}\text { A mouse-human monoclonal chimeric } \\
\text { antibody to TNF- } \alpha\end{array}$ & Multiple manifestations \\
\hline Interferon- $\alpha$ & Large class of glycoproteins known as cytokines & Multiple manifestations \\
\hline IL-23p19 antibody & Anti-interleukin-23p19-specific antibodies & Multiple manifestations \\
\hline Etanercept & $\begin{array}{l}\text { A TNF receptor-IgG fusion molecule that } \\
\text { resembles the TNF- } \alpha \text { receptor }\end{array}$ & Ocular \\
\hline Rituximab & $\begin{array}{l}\text { A chimeric monoclonal antibody to CD20 that } \\
\text { depletes the body of CD20-positive B cell }\end{array}$ & Ocular \\
\hline Trastuzumab & $\begin{array}{l}\text { A humanized murine monoclonal antibody that } \\
\text { interferes with the HER2/neu receptor }\end{array}$ & Multiple manifestations \\
\hline
\end{tabular}

Controlled data on the use of biotherapy in cardio-Behçet are almost unavailable and thus we are obliged to use data biologic agents used in other complications of $\mathrm{BD}$, and also in these conditions more controlled data are needed. However, some authors $[29,30]$ state that there is enough published experience to suggest that biotherapy may represent an important therapeutic improvement for patients with $\mathrm{BD}$ who have severe and resistant manifestations, or who are intolerant to standard immunosuppressive regimens. Considering the role of tumor necrosis factor (TNF)- $\alpha$ in triggering prothrombotic adhesion molecules, using TNF blocking agents in cardio-Behçet could be theoretically of some interest to reduce venous thrombosis relapse. A review [31] reports data from 369 patients with $\mathrm{BD}$ who were treated with biotherapy in 20 countries. Patients were inadequately controlled with, or intolerant to, other immunosuppressive regimens, including interferon; 20 patients received more than 1 anti-TNF agent, they were mostly treated with infliximab, but also with etanercept and adalimumab; patients were treated for resistant mucocutaneous, ocular, gastrointestinal, and central nervous system involvement (results from neuro-Behçet should be useful for the therapy of cardio-Behçet because of the similarity in vascular lesions). The authors conclude that there is enough published experience to suggest that TNF blockade represents an important therapeutic advancement for patients with severe and resistant, or intolerant, to standard immunosuppressive regimens but in many cases the use of TNF-antagonists in thrombosis of BD was disappointing especially with regard to potential prothrombotic activities and it has also found the predisposition to frequent autoantibody formation, notably anticardiolipin [32].

Two case-report papers report the use of infliximab, combined with endovascular intra-aortic stenting, in treating aortitis and 2 cases of pulmonary artery aneurysm with intra-cardiac thrombosis, 1 case with femoral profunda artery aneurysm and 2 cases with magic syndrome; the therapy induced more than a year of complete remission [33, 34]. On the other hand, studies on infliximab with a sufficient number of patients with BD deal with ocular manifestations [34, 35]. Infliximab has been used for 6 years on 50 patients with BD with posterior uveitis [34], and it was rapidly effective; mild infusion reactions were recorded in $18 \%$ of patients, urinary infections in $12 \%$, upper airways infections in $16 \%$, and slight liver enzymes in $4 \%$ with no severe adverse-event requiring infliximab interruption; lastly, antinuclear antibodies positivity was 
observed in $20 \%$ of patients during the whole period of follow-up, however, no patients developed signs or symptoms of lupus- like syndrome. The other paper [35] describes the effects of interferon- $\alpha$, infliximab, etanercept, and rituximab in the treatment of ocular manifestations of patients with BD (there are no cases with cardio-Behçet) who responded poorly to conventional therapies: interferon- $\alpha$ was beneficial in the therapy of uveitis and many patients enjoyed durable remissions of their ocular inflammatory disease even after discontinuation of therapy, but side-effects were almost universal and some were dangerous. Infliximab was the most extensively studied biologic agent: it was rapidly effective in many cases of uveitis, though with conflicting data concerning the ability to induce durable remission after cessation of treatment, and adverse-effects were relatively rare but some were serious and their list is impressive [37]. A review [38] describes the use of infliximab in neuro-Behçet and the data might be important for cardio-Behçet: the results were not as promising as preliminary reports would indicate, and confirm the declining therapeutic effect of infliximab in long-term use.

The use of other biologic agents in BD is even less documented. Etanercept had little positive reports in patients with uveitis [35] but there are no data on vasculo-Behçet. Rituximab was effective in treating microscopic polyangiitis, Wegener's granulomatosis, and antineutrophil cytoplasmic antibodies-associated renal vasculitis [39] and it might be effective in vasculo-Behçet. However, the use of rituximab in cardio-Behçet may be problematic because the infusion induces many side-effects in at least $20 \%$ of patients (to reduce their severity prior to the infusion of rituximab patients should receive acetaminophen, an antihistamine drug and sometimes also methylprednisolone) and although these symptoms are usually modest, cardiovascular adverse-effects of rituximab have included myocardial infarction, ventricular fibrillation, and cardiogenic shock (as parts of an infusion related reaction) and hypotension in 10\% of cases [36-39].

In 17 patients with $\mathrm{BD}$, infliximab lost efficacy and was replaced with adalimumab (the main manifestations requiring switching were the mucocutaneous lesions, followed from retinal vasculitis and neurological involvement); 9 patients showed sustained remission and 3 a good response; no side-effects were observed [40]. Thus is seems that patients with $\mathrm{BD}$ who show a scarce response or adverse-events to infliximab may be successfully treated with adalimumab, but the number of treated patients is small and it is premature to say if the efficacy shall last.
Always searching for better therapies, in vitro experiments showed that interferon- $\alpha$ could inhibit interleukin-17 expression and increased interleukin- 10 production by PBMCs and CD4+ T-cells and a study reports that the drug was effective in the in the therapy of uveitis in patients with BD [41] and also in the therapy of gastrointestinal manifestations of BD [42]. However, interferon- $\alpha$ induces many side-effects and there are no published data about its use in cardio-Behçet.

The human anti-interleukin-17-receptor monoclonal antibody brodalumab was successfully used in the therapy of psoriasis [43] and unpublished data confirm its use in in the therapy of severe mucocutaneous manifestation of BD.

Interleukin-23 is a member of the interleukin- 12 cytokine family that drives a highly pathogenic T-cell population involved in the initiation of autoimmune diseases; anti-interleukin-23p19-specific antibodies inhibited multiple inflammatory pathways that are critical for driving autoimmune central nervous system inflammation [44]. It is likely that anti-interleukin-23 antibodies will be used in the therapy of neuro-Behçet. Of course, it is unclear if the therapy will be useful in cardio-Behçet.

Immunotherapy is considered for the therapy of serious manifestations of BD but there are problems with its efficacy and safety. The number of presently used biologic agents is already large and newer drugs are continuously introduced. Bevacizumab is an unpleasant candidate because it has been found to increase platelet aggregation and adhesion to the vascular endothelium [45]. Imatinib, another biologic agent, has a proven cardiac toxicity and may induce congestive heart failure [46]. Searching for a better alternative for infliximab, tocilizumab was used to treat severe cases of neuro-Behçet [47] but it may be responsible for cardiac toxicity [48].

Interestingly, the way of administration of biologic agents may play an important clinical role because the subcutaneous use of abatacept induced less side-effects than the intravenous therapy [49].

\section{Conclusions}

$\mathrm{BD}$ is an enigmatic inflammatory disorder with multisystemic complications which is endemic in some countries but can be seen in the entire world. Valid diagnostic criteria are available. The pathology is related to a specific perivasculitis with involvement of both arteries and veins of all sizes. 
Minor arterial and cardiac involvement is frequent in $\mathrm{BD}$ but is usually asymptomatic. In exceptional cases cardiac symptoms may be the $1^{\text {st }}$ manifestation of BD. The prevalence of severe cardiac complications (cardio-Behçet) should be $<10 \%$. Impressive therapeutic improvement has been achieved by using appropriate catheterization techniques, coronary and intra-arterial stents, colchicine, drug-response modifying drugs and immunotherapy, but, still, cardio-Behçet has a poor prognosis. Efforts are undertaken to improve morbidity and prognosis with the use of newer drugs. An important part of the complications in $\mathrm{BD}$ are related to the frequent thromboembolic complications and there is a high possibility that newer oral anticoagulants will be superior to the classical anticoagulants presently used. Available biologic agents have already been frequently used and seem to have improved the prognosis, but efforts are undertaken to find newer biologic agents with better therapeutic performance and less side-effects.

Summarizing the effects of the presently used biotherapy in BD as much as possible, interferon- $\alpha$ is effective against many ocular, genital and, perhaps, vascular manifestations, but its effectiveness is limited by frequent adverse-effects (even if not dangerous for the cardiovascular system). Infliximab is a valid option in the therapy of ocular and cutaneous manifestations but is less convincing in the therapy of vascular manifestations in vascular- and neuro-Behçet; furthermore, side-effects, including severe cardiovascular complications, are seen in a minority of patients; perhaps worse, infliximab seems to loose efficacy in long-term therapy, while pharmacogenetics and receptor polymorphism may explain the existence of non-responders and the occurrence of resistance. Adalimumab might be a promising alternative for infliximab and seems to exert a good effect in aneurysmatic and other vascular complications. However, we lack long-term studies. Other biologic agents have been used only in few cases and it is too early to say if they offer new therapeutic perspectives.

\section{Acknowledgements}

The authors are grateful to Mrs. J. Bugmann and Mrs. H. Brogli for their assistance and secretarial help.

\section{Conflict of interest: none declared}

\section{References}

1. Cocco G, Gasparyan YA. Behçet's disease: An Insight from a cardiologist's point of view. The Open Cardiovasc Med J, 2010; 4: $63-70$.
2. Zouboulis CC, Kaklamanis P. Early descriptions of Adamantiades-Behçet's disease. Ann Rheum Dis, 2003; 62: 691-692.

3. James DG. 'Silk route disease' (Behçet's disease). West J Med, 1988; 148: 433-437.

4. Saadoun D, Wechsler B. Behcet's disease. Orphanet J Rare Diseases, 2012; 7: 20.

5. Fereydoun D. Diagnosis/classification criteria for Behçet's disease. Hindawi Pub Corp; Pathol Res Internat 2012; ID: 607921; doi:10.1155/2012/607921.

6. International Team for the Revision of the International Criteria for Behcet'sDisease, Revision of the International Criteria for Behçet'sdisease (ICBD), ClinExperimRheumat, 2006; 24: S14-S15.

7. Owlia MB, Mehrpoor G. Behçet'sdisease: New concepts in cardiovascular involvements and future direction for treatment. ISRN Pharmacology, 2012; ID: 760484; doi:10.5402/2012/ /760484.

8. Haghighi AB, Sharifzad HR, Matin S, Rezaee S. The pathological presentations of neuro-Behçet disease: a case report and review of the literature. Neurologist, 2007; 13: 209-214.

9. Hasan A, Fortune F, Wilson A et al. Role of gamma delta T cells in pathogenesis and diagnosis of Behçet's disease. Lancet, 1996; 347: 789-794.

10. Ekinci NS, Alpsoy E, Karakas AA et al. IL-17A has an important role in the acute attacks of Behçet's disease. J Invest Dermatol, 2010; 130 : 2136-2138.

11. Gerstzen RE, Tager AW. The monocyte in atherosclerosis: Should I stay or should I go now? New Engl J Med, 2012; 366: 1734-1736.

12. Khan JA, Cao M, Kang BY et al. Systemic human netrin-1 gene delivery by adeno-associated virus type 8 alters leukocyte accumulation and atherogenesis in vivo. Gene Ther, 2011; 18: 437-444.

13. Kiraz S, Ertenli I, Oztürk MA et al. Pathological haemostasis and 'prothrombotic state' in Behçet's disease. Thromb Res, 2002; 105: 25-33.

14. Yagmur J, Sener S, Acikgoz N et al. Subclinical left ventricular dysfunction in Behçet's disease assessed by two-dimensional speckle tracking echocardiography. Eur J Echocardiogr, 2011; 12: 536-541.

15. Manfrini O, Xhyheri B, Pizzi C. Acute coronary syndrome and Behçet's disease. J Cardiovasc Med, 2012; 13: 401-402.

16. Aktürk E, Yağmur J, Kurtoğlu E et al. Left atrial volume and function in patients with Behçet's disease assessed by real-time three-dimensional echocardiography. Eur J Echocardiogr, 2012; 16: 650-655.

17. Candan I, Erol C, Sonel A, Akalin H. Behçet's disease: Cardiac and pulmonary involvement. Eur Heart J, 1986; 7: 999-1002 .

18. Koç Y, Güllü I, Akpek G et al. Vascular involvement in Behçet's disease. J Rheumatol, 1992; 19: 402-410.

19. Lee I, Park S, Hwang I, Kim M-J et al. Cardiac Behçet disease presenting as aortic valvulitis/aortitis or right heart inflammatory mass: Aclinicopathologic study of 12 cases. Am J SurgPathol, 2008; 32: 390-398.

20. Cevik C, Otahbachi M, Nugent K et al. Coronary artery aneurysms in Behçet's disease. CardiovascRevasc Med, 2009; 10: 128-129.

21. Calamia K, Schirmer M, Melikoglu M. Major vessel involvement in Behçet's disease: An update. Curr Opinion Rheumatol, 2011; 23: 24-31.

22. Geri G, Wechsler B, ThiHuong du L et al. Spectrum of cardiac lesions in Behçet disease: A series of 52 patients and review of the literature. Medicine (Baltimore), 2012; 91: 25-34. 
23. Saadoun D, Asli B, Wechsler B et al. Long-term outcome of arterial lesions in Behçet disease: A series of 101 patients. Medicine (Baltimore), 2012; 91: 18-24.

24. Kingston M, Ratcliffe JR, Alltree M, Merendino KA. Aneurysm after arterial puncture in Behçet's disease. Br Med J, 1979; 1: 1766-1167

25. Cocco G, Chu DCC, Pandolfi S. Colchicine in clinical medicine. A guide for internists. Eur J Int Med, 2010; 21: 503-508.

26. Gasparyan AY, Ayvazyan L, Cocco G, Kitas GD. Adverse cardiovascular effects of antirheumatic drugs: Implications for clinical practice and research. Curr Pharmaceutical Design, 2012; 18: 1543-1555.

27. Handschin C, Meyer UA. Warum reagiert mein patient anders auf dieses medikament? Schweiz Med Forum, 2012; 12: 425-433 .

28. www.vfa.de/personalisiert.

29. Alexoudi I, Kapsimali V, Vaiopoulos A et al. Evaluation of current therapeutic strategies in Behçet's disease. ClinRheumatol, 2011; 30: 157-163.

30. Köse O. Development of immunopathogenesis strategies to treat Behçet's disease. Patholog Res Int, 2012; 2012: 261989 [Epub 2012 Apr 3].

31. Fragiadaki K, Giavri E, Sfikakis PP. Anti-TNF agents for Behçet'sdisease: Analysis of published data on 369 patients. Seminars Arthritis Rheumatism, 2011: 41: 61-70.

32. Atzeni F, Sarzi-Puttini P. Autoantibody production in patients treated with anti-TNF-alpha. Expert Rev ClinImmunol, 2008; 4: 275-280.

33. Hidalgo-Tenorio C, Sabio-Sánchez JM, P. Linares PJP et al. Magic syndrome and true aortic aneurysm. ClinRheumat, 2008; 27: 115-117.

34. Lee MA, Kyle S, Moots RJ. The successful treatment of inflammatory aortitis in Behçet's disease with Infliximab and endovascular intra-aortic stenting. Rheumatol Reports, 2012; 4; doi:10.4081/rr.2012.e2.

35. Benitah NR, Sobrin L, George Papaliodis GN. The use of biologic agents in the treatment of ocular manifestations of Behçet'sdisease. Seminars Ophthalmol, 2011; 26: 295-303.

36. Yamada Y, Sugita S, Tanaka H et al. Timing of recurrent uveitis in patients with Behçet's disease receiving infliximab treatment. Br J Ophthalmol, 2011; 95: 205-208.

37. www.drugs.com/sfx/rituximab-side-effects.html.
38. Haghighi BA, Safari A, Nazarinia MA, Habibagahi Z, Shenavandeh S. Infliximab for patients with neuro-Behçet's disease: Case series and literature review. ClinRheumatol, 2011; 7: 1007-1012.

39. Jones RB, Cohen Tervaert JW, Hauser T et al.; for the European Vasculitis Study Group. Rituximab versus cyclophosphamide in ANC-associated renal vasculitis. N Engl J Med, 2010; 363: 211-220.

40. Olivieri I, Leccese P, D'Angelo S et al. Efficacy of adalimumab in patients with Behçet's disease unsuccessfully treated with infliximab. ClinExpRheumatol, 2011; 29 (Suppl. 67): S54-S57.

41. NilüferYalçindağ F, Aslihan Uzun A. IFN-alpha-2a treatment is effective in Behçet uveitis refractory to traditional treatment regimens and provides significant improvement in visual prognosis. J Ocular PharmacolTherap, doi:10.1089/jop.2011.0238[ahead of print].

42. Itaru I; Seiichi K. Anti-tumor necrosis factor monoclonal antibody therapy for intestinal Behçetdisease in an adolescent. J PedGastroenter Nutrition, 2011; 53: 686-688.

43. Papp KA, Leonardi C, Menter A et al.Brodalumab, an anti-interleukin-17-receptor antibody for psoriasis. New Engl J Med, 2012; 366: 1181-1189.

44. Yi Chen Y, Langrish CL, Mckenzie B et al. Anti-IL-23 therapy inhibits multiple inflammatory pathways and ameliorates autoimmune encephalomyelitis. J Clin Invest, 2006; 116: 1317-1326.

45. Pereg D, Lishner M. Bevacizumab treatment for cancer patients with cardiovascular disease: a double edged sword? Eur Heart J, 2008; 29: 2325-2326.

46 Kerkelä R, Grazette L, Yacobi R et al. Cardiotoxicity of the cancer therapeutic agent imatinibmesylate. Nat Med, 2006; 12: 908-916.

47. Shapiro LS, Farrell J, Haghighi AB. Tocilizumab treatment for neuro-Behçet's disease, the first report. ClinNeurolNeurosurg, 2012; 3: 297-298.

48. Baselga J, Lynn Smith B, Rafterty EA, Mambonaty A. Case 16-2012: A 32-year-old woman with HER2-positive brestcancer. New Engl J Med, 2012; 366: 2018-2026.

49. Genovese MC, Becker JC, Schiff M et al. Subcutaneous abatacept versus intravenous abatacept: A phase IIIb non inferiority study in patients with an inadequate response to methotrexate. Arthritis Rheum, 2011; 63: 2854-2864. 